\title{
ON ASYMPTOTIC VALUES OF CERTAIN SETS OF ATTACHED PRIME IDEALS
}

\author{
by A.-J. TAHERIZADEH
}

(Received 11 May, 1987)

Introduction. In his paper [1], M. Brodmann showed that if $M$ is a finitely generated module over the commutative Noetherian ring $R$ (with identity) and $a$ is an ideal of $R$ then the sequence of sets $\left\{\operatorname{Ass}\left(M / \mathfrak{a}^{n} M\right)\right\}_{n \in \mathbb{N}}$ and $\left\{\operatorname{Ass}\left(\mathfrak{a}^{n-1} M / \mathfrak{a}^{n} M\right)\right\}_{n \in \mathbb{N}}$ (where $\mathbb{N}$ denotes the set of positive integers) are eventually constant. Since then, the theory of asymptotic prime divisors has been studied extensively: in [5, Chapters 1 and 2], for example, various results concerning the eventual stable values of $\operatorname{Ass}\left(R / \mathfrak{a}^{n}\right)$ and Ass $\left(a^{n-1} / \mathfrak{a}^{n}\right)$, denoted by $A^{*}(\mathfrak{a})$ and $B^{*}(a)$ respectively, are discussed. It is worth mentioning that the above mentioned results of Brodmann still hold if one assumes only that $A$ is a commutative ring (with identity) and $M$ is a Noetherian $A$-module, and $\operatorname{Ass}_{A}(M)$, in this situation, is regarded as the set of prime ideals belonging to the zero submodule of $M$ for primary decomposition.

Recently, in [7], Sharp proved a result which is, in a sense, dual to the above mentioned theorem of Brodmann. He showed [7, Theorem (3.1)] that if $M$ is an Artinian module over the commutative ring $A$ (with identity) then $\operatorname{Att}_{A}\left(0:_{M} \mathfrak{a}^{n}\right)$ and $\operatorname{Att}_{A}\left[\left(0:_{M} \mathfrak{a}^{n}\right) /\left(0:_{M} \mathfrak{a}^{n-1}\right)\right]$ are, for all sufficiently large $n$, independent of $n$, that is, the sequences of sets $\left\{\operatorname{Att}_{A}\left(0:_{M} a^{n}\right)\right\}_{n \in \mathbb{N}}$ and $\left\{\operatorname{Att}_{A}\left[\left(0:_{M} a^{n}\right) /\left(0:_{M} a^{n-1}\right)\right]\right\}_{n \in \mathbb{N}}$ are ultimately constant; we denote these eventual stable values by $\mathrm{At}^{*}(\mathfrak{a}, M)$ and $\mathrm{Bt}^{*}(\mathfrak{a}, M)$ respectively (following McAdam [5]).

Now it is natural to ask whether there are, for Artinian modules over a commutative ring, results dual to those discussed in [5, Chapters 1 and 2]. However, although some of the techniques which can be used in the Noetherian case are not obviously available here, we have been able to obtain partial results.

Again let $R$ be a Noetherian ring and $\mathfrak{a}$ an ideal of $R$ and let $T$ be an indeterminate. In [5, Proposition 1.15] a characterization of $B^{*}(a)$ in terms of the Rees ring of $R$ with respect to $a$, that is, the ring $R^{\prime}=R\left[a T, T^{-1}\right]$, is given. Also in the same book (Proposition 2.3), it turns out that $\mathfrak{p} \in A^{*}(\mathfrak{a}) \backslash B^{*}(\mathfrak{a})$ if and only if there is $k \in \mathbb{N}$ such that $\mathfrak{p}^{(k)}$, the $k$ th symbolic power of $\mathfrak{p}$, is a component of a primary decomposition of $\mathfrak{a}^{n}$ for all sufficiently large $n$.

Now let $M$ be an Artinian module over the commutative ring $A$. The purpose of this paper is to provide, using the Artinian property of the $A$-module $M$, results dual to those mentioned in the previous paragraph.

This note is divided into three sections. In Section 1, some notation and preliminary results about the theory of secondary representation are given.

In Section 2, a characterization of $\mathrm{Bt}^{*}(a, M)$ (where $\mathfrak{a}$ is finitely generated) in terms of certain prime ideals of the (small) Rees ring of $A$ with respect to a, that is, the ring $R:=A[a T]$ ( $T$ an indeterminate), is given.

Glasgow Math. J. 30 (1988) 293-300. 
Finally, in Section 3 we prove the following result: let $M$ be an Artinian module over the commutative ring $A$ and $\mathfrak{a}$ and $\mathfrak{p}$ ideals of $A$ such that $\mathfrak{p}$ is prime. Suppose, further, that $S=A \backslash \mathfrak{p}$, a multiplicatively closed subset of $A$, and denote the submodule $\bigcap_{x \in S} x M$ of $M$ by $S(M)$ (see Lemma (1.3) for the connection between this submodule and secondary submodules of $M)$. Then $\mathfrak{p} \in \operatorname{At}^{*}(\mathfrak{a}, M) \backslash \mathrm{Bt}^{*}(\mathfrak{a}, M)$ if and only if there is $k \in \mathbb{N}$ such that, for all sufficiently large $n, S\left(0:_{M} \mathfrak{p}^{k}\right)$ is a term of a reduced secondary representation for $\left(0:_{M} \mathfrak{a}^{n}\right)$ (for definition of reduced secondary representation see Section 1(ii) below).

1. Notation and preliminary results about the theory of secondary representation. Throughout the paper, $A$ will denote a commutative ring (with non-zero identity), $M$ will denote an $A$-module and a will denote an ideal of $A$. We use $\mathbb{Z}$ to denote the set of integers and $\mathbb{N}$ to denote the set of positive integers. If $M$ is Artinian over $A$ then we denote the eventual stable values of $\operatorname{Att}\left(0:_{M} a^{n}\right)$ and $\operatorname{Att}_{A}\left[\left(0:_{M} a^{n}\right) /\left(0:_{M} a^{n-1}\right)\right]$ by $\mathrm{At}^{*}(\mathfrak{a}, M)$ and $\mathrm{Bt}^{*}(\mathfrak{a}, M)$ respectively.

The theory of secondary representation is discussed in $[4,3,6]$; however, we shall use the terminology of [4] about this theory and recall the following from [4].

(i) The $A$-module $M$ is said to be secondary if $M \neq 0$ and, for each element $a$ of $A$, the endomorphism of $M$ given by multiplication by $a$ is either surjective or nilpotent. If $M$ is secondary then $\sqrt{ }(0: M)$ is a prime ideal, say $\mathfrak{p}$, and then $M$ is said to be $\mathfrak{p}$-secondary.

(ii) A secondary representation of an $A$-module $M$ is an expression for $M$ as a sum of secondary submodules, say,

$$
M=N_{1}+\ldots+N_{r}
$$

The representation (*) is said to be reduced (or minimal) if

(a) the prime ideals $\mathrm{V}\left(0: N_{i}\right)(1 \leqq i \leqq r)$ are distinct, and

(b) for $1 \leqq i \leqq r, N_{i} \nsubseteq \sum_{\substack{j=1 \\ j \neq i}}^{r} N_{j}$.

Any secondary representation of $M$ can be refined to a reduced one. If $M$ has a secondary representation, we shall say $M$ is representable.

A few facts from [4] will be essential in the argument below, and we state them, without proof, for convenience.

Throughout Theorem 1.1-Proposition $1.5, M$ will be a representable $A$-module and

$$
M=N_{1}+\ldots+N_{r}
$$

will be a reduced secondary representation with $\sqrt{ }\left(0: N_{i}\right)=\mathfrak{p}_{i}(1 \leqq i \leqq r)$.

First Uniqueness Theorem 1.1 [4, (2.2)]. The set of prime ideals $\left\{\mathfrak{p}_{1}, \ldots, \mathfrak{p}_{r}\right\}$ depends only on $M$ and not on the minimal secondary representation. More precisely, the following are equivalent:

(1) $\mathfrak{p}$ is one of $\mathfrak{p}_{i}(1 \leqq i \leqq r)$;

(2) $M$ has a p-secondary quotient module;

(3) $M$ has a quotient $Q$ such that $\sqrt{ }(0: Q)=\mathfrak{p}$; 
(4) $M$ has a quotient $Q$ such that $\mathfrak{p}$ is minimal in the set of prime ideals containing $\operatorname{Ann}(Q)$.

Definition 1.2. The prime ideals $\mathfrak{p}_{1}, \ldots, \mathfrak{p}_{r}$ are called the attached primes of the representable $A$-module $M$, and the set which they form is denoted by $\operatorname{Att}_{A}(M)$ or $\operatorname{Att}(M)$.

Lemma 1.3 [4, (3.1)]. Let $S$ be a multiplicatively closed subset of $A$. Suppose that the attached primes $\mathfrak{p}_{i}$ are numbered so that $S \cap \mathfrak{p}_{i}=\varnothing$ for $i=1, \ldots, r^{\prime}$ and $S \cap \mathfrak{p}_{i} \neq \varnothing$ for $i=r^{\prime}+1, \ldots, r$. Then the following submodules of $M$ are equal:

(1) $\bigcap_{x \in S} x M$

(2) $\sum_{i=1}^{r^{\prime}} N_{i}$,

(3) the sum of all $\mathfrak{p}$-secondary submodules $N$ of $M$ such that $\mathfrak{p} \cap S=\varnothing$.

Definition 1.4 (see [4, (3.1)]). Let $S$ be a multiplicatively closed subset of $A$. The submodule defined in Lemma 1.3 is denoted by $S(M)$, and so

$$
S(M)=\bigcap_{x \in S} x M .
$$

Proposition 1.5 [4, (3.4)]. Let $S$ be a multiplicatively closed subset of $A$. Then $S(M)=a M$ for some $a \in S$.

PROPOSITION 1.6 [4, (4.1)]. If $N$ is a representable submodule of $M$ then

$$
\operatorname{Att}(M / N) \subseteq \operatorname{Att}(M) \subseteq \operatorname{Att}(N) \cup \operatorname{Att}(M / N)
$$

TheORem 1.7 (see [4, (5.2)]). Every Artinian A-module has a secondary representation and hence a reduced secondary representation.

2. A characterization of $\mathrm{Bt}^{*}(a, M)$. Before proceeding to the desired characterization of $\mathrm{Bt}^{*}(a, M)$, we need two lemmas which will be given next.

LeMma 2.1. Let $A=\bigoplus_{n \in \mathbb{Z}} A_{n}$ be a graded ring and $\mathfrak{p}_{1}, \ldots, \mathfrak{p}_{n}$ homogeneous prime ideals of $A$ such that $A A_{1} \ddagger \bigcup_{i=1}^{n} \mathfrak{p}_{i}$. Then there exists an integer $h_{0}$ such that, for all $h \geqq h_{0}$, $A_{h} \nsubseteq \bigcup_{i=1}^{n} \mathfrak{p}_{i}$.

Proof. We may assume that for each pair $i \neq j, 1 \leqq i, j \leqq n$, there exists an integer $h_{i j}$ such that $\mathfrak{p}_{i} \cap A_{h_{i j}} \notin \mathfrak{p}_{j} \cap A_{h_{i j}}$. For each pair $i \neq j, 1 \leqq i, j \leqq n$, choose a homogeneous element $a_{i j}$ so that $a_{i j} \in \mathfrak{p}_{i} \cap A_{h_{i j}} \backslash \mathfrak{p}_{j} \cap A_{h_{i j}}$. Set $a_{j}:=\prod_{\substack{i=1 \\ i \neq j}}^{n} a_{i j}(1 \leqq j \leqq n)$. Then $a_{j} \in\left(\bigcap_{\substack{i=1 \\ i \neq j}}^{n} \mathfrak{p}_{i}\right) \backslash \mathfrak{p}_{j}$ $(1 \leqq j \leqq n)$. Let the degree of $a_{j}$ be $\delta_{j}(1 \leqq j \leqq n)$ and $\delta:=\max \left\{\delta_{1}, \ldots, \delta_{n}\right\}$. 
Next since $A A_{1} \nsubseteq \mathfrak{p}_{j}(1 \leqq j \leqq n), A_{1} \nsubseteq \mathfrak{p}_{j}(1 \leqq j \leqq n)$. So, for each $j(1 \leqq j \leqq n)$ and for any $n^{\prime}(\geqq 1)$, there exists a homogeneous element $b_{n^{\prime}}^{j}$ of degree $n^{\prime}$ such that $b_{n^{\prime}}^{j} \notin \mathfrak{p}_{j}$. Put $h_{0}=\delta+1$. Then, for all $h \geqq h_{0}$, the homogeneous element $c:=\sum_{j=1}^{n} a_{j} b_{h-\delta_{j}}^{j}$ is of degree $h$ and $c \notin p_{j}$.

Remark. I owe this lemma to Mr R. Enshaei, who pointed it out to me.

In the remainder of the paper, $M$ will denote an Artinian A-module.

For the next lemma, suppose that $a=\left(a_{1}, \ldots, a_{s}\right)$ and $T$ is an indeterminate. Further, suppose that $R:=A\left[a_{1} T, \ldots, a_{s} T\right]$ is the subring of $A[T]$ generated by $A$ and $a_{1} T, \ldots, a_{s} T$ and called the (small) Rees ring of $A$ with respect to $a$ and graded in the usual way by $\mathbb{Z}$. Set $G=\bigoplus_{n \in \mathbb{Z}} G_{n}$, where, for $n \in \mathbb{Z}$,

$$
G_{n}= \begin{cases}0 & \text { if } n>0 \\ \left(0:_{M} a^{-n+1}\right) /\left(0:_{M} a^{-n}\right) & \text { if } n \leqq 0 .\end{cases}
$$

Then we have the following lemma.

Lemma 2.2. With the same notation and assumptions as above, $G$ is an Artinian $R$-module.

Proof. Let $x_{1}, \ldots, x_{s}$ be indeterminates over $A$ and let $R^{\prime}=\bigoplus_{n \in \mathbb{Z}} R_{n}^{\prime}$ denote the ring $A\left[x_{1}, \ldots, x_{s}\right]$ graded in the usual way, so that $R_{n}^{\prime}=0$ for $n<0$. Now make $G$ into a graded $R^{\prime}$-module as in [2, p. 54]: if $n$ is an integer with $n<0, m \in\left(0::_{M} \mathfrak{a}^{-n+1}\right)$ and $1 \leqq i \leqq s$, put

$$
x_{i}\left(m+\left(0:_{M} \mathfrak{a}^{-n}\right)\right)=a_{i} m+\left(0:_{M} \mathfrak{a}^{-n-1}\right) \in G_{n+1} .
$$

By [2, Theorem 1(ii)], $G$ is an Artinian $R^{\prime}$-module.

Next there exists a surjective ring homomorphism, say, $\phi: R^{\prime} \rightarrow R$ such that $x_{i}$ $(1 \leqq i \leqq s)$ is mapped into $a_{i} T$ and $\operatorname{ker} \phi \subseteq \operatorname{Ann}_{R^{\prime}}(G)$. Thus $G$ has a structure as an $R$-module and hence $G$ is Artinian as an $R$-module by the above argument.

Definitions and Remark 2.3 (see [7, Section 2]). Let $A=\bigoplus_{n \in \mathbb{Z}} A_{n}$ and $M=\bigoplus_{n \in \mathbb{Z}} M_{n}$ a graded $A$-module.

(i) The graded $A$-module $M$ is said to be graded Artinian if $M$ satisfies the minimal conditions for homogeneous submodules.

(ii) The graded $A$-module $M$ is said to be graded-secondary if $M \neq 0$ and, for each homogeneous element $a$ of $A$, the endomorphism of $M$ given by multiplication by $a$ is either surjective or nilpotent.

In [7, Section 2], Sharp developed some ideas concerning a theory of 'graded secondary representation' of graded Artinian modules, which we shall make much use of in the next theorem. 
THEOREM 2.4. Let the notation and assumptions be as in Lemma $2: 2$ and, further, suppose that $\mathfrak{b}$ denotes the ideal $\sum_{i=1}^{s} R\left(a_{i} T\right)$ of $R$. Then $\mathfrak{p} \in \mathrm{Bt}^{*}(\mathfrak{a}, M)$ if and only if there exists $\mathfrak{q} \in \operatorname{Att}_{R}(G)$ such that $\mathfrak{b} \notin \mathfrak{q}$ and $\mathfrak{q} \cap A=\mathfrak{p}$.

Proof. We use the ideas involved in the proof of [7, Theorem (3.1)]. By Lemma 2.2, $G$ is Artinian over $R$ and so graded Artinian, and thus, by [7, Proposition (2.4)], has a reduced graded-secondary representation

$$
G=N_{1}+\ldots+N_{r}+N_{r+1}+\ldots+N_{t}
$$

where each $N_{i}$ is a graded-secondary homogeneous submodule of $M$ and $\mathcal{V}\left(0: N_{i}\right)$ is a homogeneous prime ideal $\mathfrak{q}_{i}$, say, of $R(1 \leqq i \leqq t)$. Further, suppose that the $N_{i}$ are numbered so that

$$
\begin{array}{ll}
\mathfrak{b} \nsubseteq \mathfrak{q}_{i} & \text { for } i=1, \ldots, r, \\
\mathfrak{b} \subseteq \mathfrak{q}_{i} & \text { for } i=r+1, \ldots, t .
\end{array}
$$

For $h \in \mathbb{Z}, i=1, \ldots, t$, put $N_{i, h}=N_{i} \cap G_{h}$. Then, since $\mathfrak{b}$ is a finitely generated ideal of $R$, there exists $v \in \mathbb{N}$ such that

$$
\mathfrak{b}^{2} N_{i}=0 \text { for } i=r+1, \ldots, t .
$$

Next, from the definition of the action of $R$ on $G$ and (2), we deduce that

$$
N_{i,-(v+j)}=0 \text { for all } j \in \mathbb{N} \text { and } i=r+1, \ldots, t \text {. }
$$

Thus, by [7, Proposition (2.5)],

$$
G_{-k}=N_{1,-k}+\ldots+N_{r_{,}-k} \text { for all } k>v,
$$

and each term on the right hand side is either 0 or an $A \cap \mathfrak{q}_{i}$-secondary $A$-module. Therefore, by [7, Theorem (3.1)(i) and Proposition (2.5)],

$$
\mathrm{Bt}^{*}(\mathfrak{a}, M) \subseteq\left\{A \cap \mathfrak{q}_{i}: i=1, \ldots, r\right\} .
$$

We claim that $\mathrm{Bt}^{*}(\mathfrak{a}, M)=\left\{A \cap \mathfrak{q}_{i}: i=1, \ldots, r\right\}$. Suppose the claim is false and $\mathfrak{p}_{i}:=\mathfrak{q}_{i} \cap A \notin \mathrm{Bt}^{*}(\mathfrak{a}, M)$ for some $i, 1 \leqq i \leqq r$, and look for a contradiction.

Let $l>v$ be such that, for $n>l, \operatorname{Att}_{A}\left(G_{-n}\right)=\mathrm{Bt}^{*}(\mathfrak{a}, M)$. By Lemma 2.1, there is $h_{0} \in \mathbb{Z}$ such that, for all $h \geqq h_{0}, R_{h} \ddagger \bigcup_{i=1}^{r} \mathfrak{q}_{i}$. Choose $h \geqq h_{0}$ so that $h+k>l$ for all $k \in \mathbb{N}$. Then the representation

$$
G_{-k-h}=N_{1,-k-h}+\ldots+N_{r,-k-h}
$$

can be reduced to a minimal secondary representation because $\mathfrak{p}_{i} \notin \operatorname{Att}_{A}\left(G_{-k-h}\right)$. Therefore we must have, for all $k \in \mathbb{N}, N_{1,-(k+h)} \subseteq \sum_{\substack{j=1 \\ j \neq i}}^{r} N_{j,-(k+h)}$. Let $f_{h} \in R_{h} \backslash \bigcup_{i=1}^{r} \mathfrak{q}_{i}$. Then

$$
f_{h} N_{i,-(k+h)} \subseteq \sum_{\substack{j=1 \\ j \neq i}}^{r} f_{h} N_{j,-(k+h)} .
$$


By applying $[7,2.5]$ to this, we obtain $N_{i,-k} \subseteq \sum_{\substack{j=1 \\ j \neq i}}^{r} N_{j,-k}$ and hence

and this is a contradiction.

$$
N_{i} \subseteq \sum_{\substack{j=1 \\ j \neq i}}^{r} N_{j}
$$

3. $\operatorname{At}^{*}(\mathfrak{a}, M) \backslash \mathrm{Bt}^{*}(\mathfrak{a}, M)$. For the main result of this section, we need a few preliminary lemmas which will be given below.

LeMmA 3.1. Let $M \neq 0$ and $l \in \mathbb{N}$ be such that, for all $n \geqq l$,

$$
\operatorname{Att}\left(0: \mathfrak{a}_{M}\right)=\operatorname{At}^{*}(\mathfrak{a}, M),
$$

and

$$
\operatorname{Att}\left[\left(0:_{M} a^{n+1}\right) /\left(0:_{M} a^{n}\right)\right]=\mathrm{Bt}^{*}(a, M) .
$$

Suppose, further, that $\mathfrak{p} \in \operatorname{At}^{*}(\mathfrak{a}, M) \backslash \mathrm{Bt}^{*}(\mathfrak{a}, M)$ and, for $n \geqq l$,

$$
\left(0:_{M} \mathfrak{a}^{n}\right)=S_{n}+S_{n 1}+\ldots+S_{n t}
$$

is a reduced secondary representation with $\sqrt{ }\left(0: S_{n}\right)=\mathfrak{p}$ and $\sqrt{ }\left(0: S_{n i}\right)=\mathfrak{p}_{i}(1 \leqq i \leqq t)$. Put $T_{n}:=S_{n 1}+\ldots+S_{n t}$. Then we may arrange the $S_{n i}(1 \leqq i \leqq t)$ so that, for all $n \geqq l$, $S_{n i} \subseteq S_{n+1 i}$ and after this arrangement we have, for all $n \geqq l$,

$$
T_{n+1} \supseteq T_{n} \text { and } \operatorname{Att}\left(T_{n}\right)=\operatorname{Att}\left(0:_{M} \mathfrak{a}^{n}\right) \backslash\{\mathfrak{p}\} .
$$

Moreover, for all $n \geqq l$,

$$
\left(0:_{M} \mathfrak{a}^{n}\right)=\left(0 ;_{M} \mathfrak{a}^{l}\right)+T_{n} .
$$

Proof. The first part is easy. So we prove the second part. We prove this by induction on $n \geqq l$. For $n=l$ there is nothing to prove.

Induction step. Assume (4) when $n=r \geqq l$ and consider the case where $n=r+1$. Obviously $T_{r+1}+\left(0:_{M} \mathfrak{a}^{\prime}\right) \subseteq\left(0:_{M} \mathfrak{a}^{r+1}\right)$. We show that the reverse inclusion also holds. Suppose, on the contrary, that $\left(0:_{M} \mathfrak{a}^{r+1}\right) \neq T_{r+1}+\left(0:_{M} \mathfrak{a}^{l}\right)$. So

$$
\left(0:_{M} \mathfrak{a}^{r+1}\right) /\left(T_{r+1}+\left(0:_{M} \mathfrak{a}^{l}\right)\right) \neq 0
$$

and

$$
\begin{aligned}
0 \neq\left[\left(0:_{M} \mathfrak{a}^{r+1}\right) /\left(T_{r+1}+\left(0:_{M} \mathfrak{a}^{l}\right)\right)\right] & =\left(\left(S_{r+1}+T_{r+1}\right)+\left(0:_{M} \mathfrak{a}^{l}\right)\right) /\left(T_{r+1}+\left(0:_{M} \mathfrak{a}^{l}\right)\right) \\
& =\left[S_{r+1}+\left(T_{r+1}+\left(0:_{M} \mathfrak{a}^{l}\right)\right)\right] /\left[T_{r+1}+\left(0:_{M} \mathfrak{a}^{l}\right)\right] \\
& \cong S_{r+1} /\left[\left(T_{r+1}+\left(0:_{M} \mathfrak{a}^{l}\right)\right) \cap S_{r+1}\right]
\end{aligned}
$$

which is a homomorphic image of $S_{r+1}$ and hence $\mathfrak{p}$-secondary.

Next, by the induction hypothesis,

$$
T_{r+1}+\left(0:_{M} \mathfrak{a}^{l}\right) \supseteq T_{r}+\left(0:_{M} \mathfrak{a}^{l}\right)=\left(0:_{M} a^{r}\right),
$$


and hence the $\mathfrak{p}$-secondary $A$-module $\left(0:_{M} \mathfrak{a}^{r+1}\right) /\left(T_{r+1}+\left(0:_{M} \mathfrak{a}^{l}\right)\right)$ is isomorphic to a quotient module of $\left(0:_{M} \mathfrak{a}^{r+1}\right) /\left(0:_{M} a^{r}\right)$. Therefore $\mathfrak{p} \in \mathrm{Bt}^{*}(\mathfrak{a}, M)$ by Theorem $1.1(2)$ and this is a contradiction. The proof is now complete.

Lemma 3.2. Let $M \neq 0$ and $\mathfrak{p}$ be a prime ideal of $A$ and $S=A \backslash \mathfrak{p}$. Further, suppose that for some $k \in \mathbb{N}, S\left(0:_{M} \mathfrak{p}^{k}\right) \neq 0$, where $S\left(0:_{M} \mathfrak{p}^{k}\right)$ is defined as in Definition 1.4 , that is, $S\left(0: \mathfrak{p}_{M} \mathfrak{p}^{k}\right)=\bigcap_{x \in S} x\left(0:_{M} \mathfrak{p}^{k}\right)$. Then $S\left(0:_{M} \mathfrak{p}^{k}\right)$ is a $\mathfrak{p}$-secondary submodule.

Proof. Let $N$ be a $q$-secondary submodule of $\left(0: M p^{k}\right)$ which is such that $q \cap S=\varnothing$. Then $\mathfrak{p}^{k} N=0$ and $\mathfrak{p} \subseteq \mathfrak{q}$; so $\mathfrak{p}=\mathfrak{q}$. Thus it is immediate from Lemma 1.3 that $S\left(0:_{M} \mathfrak{p}^{k}\right)$ is p-secondary.

Lemma 3.3. Let $M \neq 0$. Suppose that for $n \geqq l, \mathfrak{p} \in \operatorname{Att}\left(0:_{M} \mathfrak{a}^{n}\right)$ and $S=A \backslash \mathfrak{p}$. Then there exists $k \in \mathbb{N}$ such that $S\left(0:_{M} \mathfrak{p}^{k}\right)$ is $\mathfrak{p}$-secondary; furthermore, $S\left(0:_{M} \mathfrak{p}^{k}\right)$ contains the $\mathfrak{p}$-secondary component of $\left(0:_{M} \mathfrak{a}^{\prime}\right)$ in any reduced secondary representation of it. $\left(S\left(0:_{M} \mathfrak{p}^{k}\right)\right.$ is the same as in Lemma 3.2.)

Proof. Suppose that $\left(0:{ }_{M} a^{l}\right)=S_{l}+S_{l 1}+\ldots+S_{l t}$ is a reduced secondary representation with $S_{l} \mathfrak{p}$-secondary. By [2, Lemma 3$]$, there exists a finitely generated ideal $\mathfrak{b}$ such that $\mathfrak{b} \subseteq \mathfrak{p}$ and $\left(0:{ }_{M} \mathfrak{b}^{n}\right)=\left(0:{ }_{M} \mathfrak{p}^{n}\right)$ for all $n \in \mathbb{N}$. Thus, since $\sqrt{ }\left(0: S_{l}\right)=\mathfrak{p}$ and $\mathfrak{b}$ is finitely generated, there is $k \in \mathbb{N}$ such that

$$
S_{l} \subseteq\left(0:_{M} \mathfrak{p}^{k}\right)
$$

Also by Proposition 1.5 , there is $a \in S$ so that $S\left(0:_{M} \mathfrak{p}^{k}\right)=a\left(0:_{M} \mathfrak{p}^{k}\right)$ and so, in view of (5), we have

$$
a S_{l}=S_{l} \subseteq S\left(0:_{M} p^{k}\right)
$$

So $S\left(0::_{M} \mathfrak{p}^{k}\right) \neq 0$ and hence, by Lemma $3.2, S\left(0:_{M} \mathfrak{p}^{k}\right)$ is $\mathfrak{p}$-secondary. The last assertion is (6).

Now we prove the result which was promised earlier.

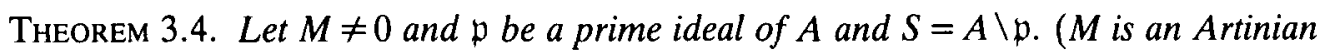
$A$-module and $\mathfrak{a}$ is a proper ideal of $A$.) Then $\mathfrak{p} \in \mathrm{At}^{*}(\mathfrak{a}, M) \backslash \mathrm{Bt}^{*}(\mathfrak{a}, M)$ if and only if there exists $k \in \mathbb{N}$ such that for all sufficiently large $n, S\left(0:_{M} \mathfrak{p}^{k}\right)$ is a term of a reduced secondary representation for $\left(0:_{M} \mathfrak{a}^{n}\right)$, where $S\left(0:_{M} \mathfrak{p}^{k}\right)$ is the same as in Lemma 3.2.

Proof. $(\Rightarrow)$ Suppose that $\mathfrak{p} \in \operatorname{At}^{*}(\mathfrak{a}, M) \backslash \mathrm{Bt}^{*}(\mathfrak{a}, M)$ and suppose that $l$ is as in Lemma 3.1. Let $\operatorname{At}^{*}(\mathfrak{a}, M)=\left\{\mathfrak{p}, \mathfrak{p}_{1}, \ldots, \mathfrak{p}_{t}\right\}$ and, for $n \geqq l$, suppose that

$$
\left(0:{ }_{M} \mathfrak{a}^{n}\right)=S_{n}+S_{n 1}+\ldots+S_{n t}
$$

is a reduced secondary representation with $\sqrt{ }\left(0: S_{n}\right)=\mathfrak{p}$ and $\sqrt{ }\left(0: S_{n i}\right)=\mathfrak{p}_{i}(1 \leqq i \leqq t)$. By Lemma 3.1, for $n \geqq l,\left(0:_{M} a^{n}\right)=\left(0:_{M} a^{l}\right)+T_{n}$, where $T_{n}$ is a submodule of $\left(0:_{M} \mathfrak{a}^{n}\right)$ such that $T_{n+1} \supseteq T_{n}$ and $\operatorname{Att}\left(T_{n}\right)=\operatorname{Att}\left(0:_{M} \mathfrak{a}^{n}\right) \backslash\{\mathfrak{p}\}(n \geqq l)$. By Lemma 3.3, there is $k \in \mathbb{N}$ such that $S_{l} \subseteq S\left(0:_{M} \mathfrak{p}^{k}\right)$ and $S\left(0:_{M} \mathfrak{p}^{k}\right)$ is $\mathfrak{p}$-secondary. 


\section{A.-J. TAHERIZADEH}

Now let $n \geqq \max \{k, l\}$. Then, by Lemma 3.1,

$$
\begin{aligned}
\left(0:{ }_{M} \mathfrak{a}^{n}\right)=\left(0:_{M} \mathfrak{a}^{l}\right)+T_{n}=\left(S_{l}+T_{l}\right)+T_{n} & =S_{l}+\left(T_{l}+T_{n}\right) \\
& =S_{l}+T_{n} \\
& \subseteq S\left(0:_{M} \mathfrak{p}^{k}\right)+T_{n},
\end{aligned}
$$

and $S\left(0:_{M} \mathfrak{p}^{k}\right)+T_{n} \subseteq\left(0:_{M} \mathfrak{a}^{n}\right)$ obviously. Thus

$$
\left(0:_{M} \mathfrak{a}^{n}\right)=S\left(0:_{M} \mathfrak{p}^{k}\right)+T_{n} \text { for all } n \geqq \max \{k, l\} .
$$

$(\Leftarrow)$ Suppose that there is $k \in \mathbb{N}$ such that $S\left(0:{ }_{M} \mathfrak{p}^{k}\right)$ is a term of a reduced secondary representation of $\left(0:_{M} \mathfrak{a}^{n}\right)$ for sufficiently large $n$. Then $S\left(0:_{M} \mathfrak{p}^{k}\right) \neq 0$ and thus, by Lemma $3.2, \mathfrak{p}$-secondary. Let

$$
\left(0:_{M} \mathfrak{a}^{n}\right)=S\left(0:{ }_{M} \mathfrak{p}^{k}\right)+S_{n 1}+\ldots+S_{n t}(n \text { is sufficiently large })
$$

be a reduced secondary representation. Then obviously $\mathfrak{p} \in \operatorname{At}^{*}(\mathfrak{a}, M)$.

Next, we have

$$
\begin{aligned}
\left(0:_{M} \mathfrak{a}^{n+1}\right) /\left(0:_{M} \mathfrak{a}^{n}\right) & =\left(S\left(0:_{M} \mathfrak{p}^{k}\right)+S_{n+11}+\ldots+S_{n+1 t}\right) /\left(0:_{M} \mathfrak{a}^{n}\right) \\
& =\left[\left(S\left(0:_{M} \mathfrak{p}^{k}\right)+\left(0:_{M} \mathfrak{a}^{n}\right)\right) /\left(0:_{M} \mathfrak{a}^{n}\right)\right]+\sum_{j=1}^{t}\left[\left(S_{n+1 j}+\left(0:_{M} \mathfrak{a}^{n}\right)\right) /\left(0:_{M} \mathfrak{a}^{n}\right)\right],
\end{aligned}
$$

and the first term is 0 . Thus $\mathfrak{p} \notin \mathrm{Bt}^{*}(\mathfrak{a}, M)$ and hence $\mathfrak{p} \in \operatorname{At}^{*}(\mathfrak{a}, M) \backslash \mathrm{Bt}^{*}(a, M)$. The proof is now complete.

\section{REFERENCES} 16-18.

1. M. Brodmann, Asymptotic stability of Ass $\left(M / I^{n} M\right)$, Proc. Amer. Math. Soc. 74 (1979),

2. D. Kirby, Artinian modules and Hilbert polynomials, Quart. J. Math. Oxford Ser. (2) 24 (1973), 47-57.

3. D. Kirby, Coprimary decomposition of Artinian modules, J. London Math. Soc. (2) 6 (1973), 571-576.

4. I. G. Macdonald, Secondary representation of modules over a commutative ring, Symposia Mathematica 11 (1973), 23-43. 1983).

5. S. McAdam, Asymptotic prime divisors, Lecture Notes in Mathematics 1023 (Springer,

6. D. G. Northcott, Generalized Koszul complexes and Artinian modules, Quart. J. Math. Oxford Ser. (2) 23 (1972), 289-297.

7. R. Y. Sharp, Asymptotic behaviour of certain sets of attached prime ideals, J. London Math. Soc. (2) 34 (1986), 212-218.

Department of Pure Mathematics

UNIVERSITY OF SHEFFIELD

HiCKS BUILDING

SHEFFIELD S3 7RH 